\title{
Agent Chameleons: Virtual Agents Real Intelligence
}

\author{
Gregory O'Hare \\ University College Dublin \\ Brian Duffy \\ University College Dublin \\ Bianca Schoen-Phelan \\ Technological University Dublin, bianca.schoenphelan@tudublin.ie
}

See next page for additional authors

Follow this and additional works at: https://arrow.tudublin.ie/scschcombk

Part of the Computer Sciences Commons

\section{Recommended Citation}

O'Hare, G., Duffy, B., Schoen-Phelan, B., Martin, A. \& Bradley, J. (2003). Agent Chameleons: Virtual Agents Real Intelligence. In Intelligent Virtual Agents, Springer, pp.218-225.

This Book Chapter is brought to you for free and open access by the School of Computer Sciences at ARROW@TU Dublin. It has been accepted for inclusion in Books/Book Chapters by an authorized administrator of ARROW@TU Dublin. For more information, please contact arrow.admin@tudublin.ie, aisling.coyne@tudublin.ie,gerard.connolly@tudublin.ie.

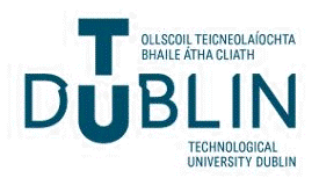


Authors

Gregory O'Hare, Brian Duffy, Bianca Schoen-Phelan, Alan Martin, and John Bradley`

This book chapter is available at ARROW@TU Dublin: https://arrow.tudublin.ie/scschcombk/4 


\title{
Agent Chameleons: Virtual Agents Real Intelligence
}

\author{
Gregory M.P. O’Hare ${ }^{1}$, Brian R. Duffy², Bianca Schön ${ }^{1}$, Alan N. Martin ${ }^{1}$ \\ \& John F. Bradley ${ }^{1}$ \\ ${ }^{1}$ Department of Computer Science, University College Dublin, Belfield, Dublin 4, Ireland \\ \{gregory.ohare, bianca.schoen, alan.martin, \\ john.bradley ducd.ie \\ http: //chameleon.ucd.ie \\ ${ }^{2}$ Media Lab Europe, Sugar House Lane, Bellevue, Dublin 8, Ireland \\ brdemle.ie
}

\begin{abstract}
Agent Chameleons provides virtual agents powered by real intelligence, delivering next generation autonomic entities that can seamlessly migrate, mutate and evolve on their journey between and within physical and digital information spaces.
\end{abstract}

\section{Introduction}

"You know I always thought unicorns were fabulous creatures too, although I never saw one alive before." "Well, now that we have met," said the unicorn, "If you'll believe in me, I'll believe in you."

Lewis Carrol, "Through the looking glass"

The Agent Chameleon Project strives to develop the next generation of agents, autonomic entities that can seamlessly migrate, mutate and evolve on their journey between and within physical and digital information spaces. This challenges the traditional boundaries between the physical and the virtual through the empowerment of mobile agents. Three key attributes (mutation, migration and evolution) underpin the Agent Chameleon concept.

The ultimate survival and longevity of agents is predicated by their ability to sense, react and respond to environmental change. The response can take the form of migration across a wireless network, mutation of agent form, or evolution of the agents' form and associated capabilities. The form of an agent inextricably dictates or constrains its behaviour and capabilities within a particular environment. The optimum form is very much dependent upon its world [15]. Judicious selection of appropriate forms or persona ought to empower the entity.

Participants within the Agent Chameleons experience will engage in humancomputer collaborative activities that bridge multiple diverse digital information spaces. By imbuing artificial entities, engaged in this collaboration, with knowledge of their user and the user's environment, we strive to improve the quality of the experience offered to the user. 


\section{Related Research}

The Agent Chameleons project draws on a number of major bodies of research and seeks to extend current interpretations of agent systems, virtual environments, and embodied systems (robotics). This work builds upon seminal work conducted by the Collaborative Virtual Environment (CVE) community. Notable pioneering systems that incorporate agent-based techniques include DIVA (Distributed Intelligent Virtual Agent) [24], MAVE (Multi-agent Architecture for Virtual Environments) [6], STEVE [11], Trilogy [14], AgentSalon [23], and ECHOES [16].

The realisation of evolvable characters in virtual environments will draw inspiration from such work as Synthetic Characters at MIT-Media Laboratory [3], and work on agents as synthetic characters $[1,5,21]$.

This research resonates with work undertaken within the mixed reality field with endeavours such as the Equator project $[2,4]$ and the Can You See Me Now project [10]. Although the principles of mobile agents have started to develop (i.e. mIVA [13]), few have embraced a true sense of agent mobility through information spaces.

\section{A Context for Adaptive Agents}

The Agent Chameleon project $[9,17,25]$ explicitly places enhanced demands upon the concept of context. Context is the all-encompassing term that is instantiated as situatedness, embodiment and immersion in different fields of research [9]. Context constitutes a meta-level concept, which associates the actions and perceptions of a system with its environment. The context is viewed as a triple of task or activity, the "body" and the environment. The idea of context in artificial systems now has a new dimension. In this work the specifics of the context for the Agent Chameleon, equipped with the ability to migrate between different environments, changes. It can be immersed in VR, embodied in a robot, or situated on a PC or PDA accessing the Internet or databases. In order to do this, the traditional issues regarding mind and body in AI come to the fore.

Agent Chameleons transcends the often-misused notion of embodiment in AI by emphasising the more appropriate/reflective issue of embodiment: complete adaptivity. A strong sense of intelligence requires a strong sense of embodiment, or complete embodiment. Not only does embodiment require being situated, which can be superficially or easily dealt with by providing a body or embodying an agent in the physical world and effectively requiring it to interact with the physicalities, but it also includes a sense of immersion. The Agent Chameleons project takes an alternative slant on immersion: a stronger sense of context and adaptability as realised in a seamless integration across virtual reality and the actual physical reality. That is, the agent is so immersed in the context that both physical and virtual worlds merge.

This research and the sister NEXUS project [18] seeks to extend the functionality of such an agent by developing the reference of the agent being inherently linked to our reality. For example, gestures of an avatar in a VR space are fundamentally referenced in our physical reality. Similarly, the motion of the agent across numerous 
screens is based on realising a sense of mobility in physical space. The screen where we see the avatar represents a window through which the avatar can interact with us, not uniquely a window through which we can view the virtual space as is generally understood. The primary reference is the here and now, not something in some virtual space elsewhere.

Agent Chameleons aims to deliver a framework that enhances Human Computer Interaction (HCI). Specifically, we envisage the Agent Chameleons as being a basis for the delivery of a new breed of pervasive and immersive applications.

\section{The Agent Chameleons Architecture}

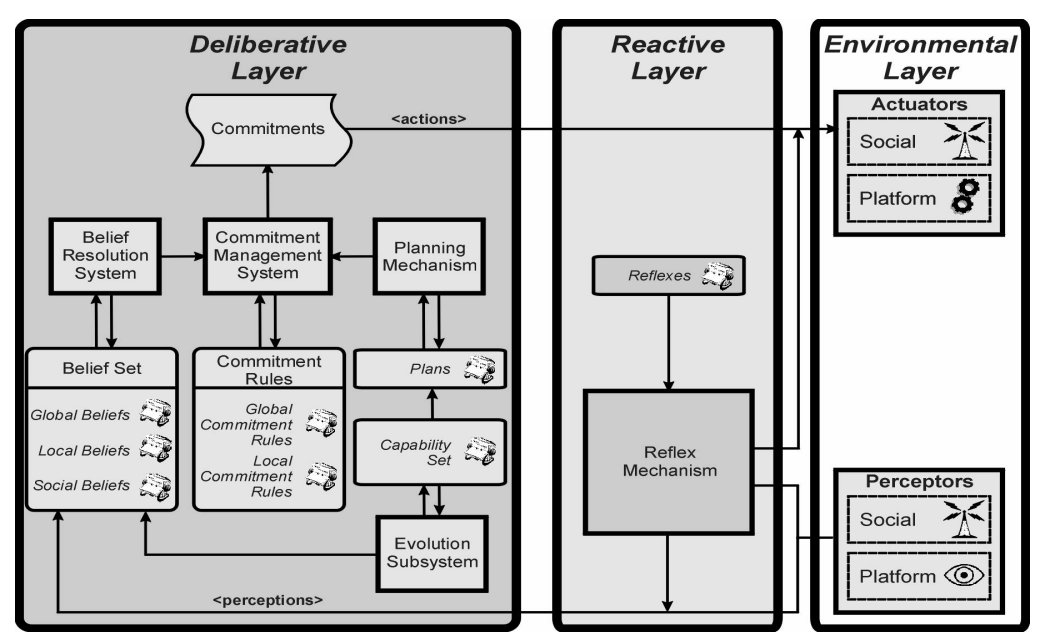

Fig. 1. Agent Chameleon Architecture

The agent architecture is comprised of three layers - Environmental, Reactive and Deliberative (see Figure 1). The Environmental Layer is responsible for the agent's low-level interaction with its environment. It consists of two types of structures Perceptors and Actuators, the former are responsible for the monitoring of the environment and the later effect the environment. The Reactive Layer provides basic reflexes empowering the agent with a collection of survival instincts. This eliminates reliance on the deliberative agent mechanisms alone. In the event that the Reactive Layer is unable to deal with a situation, the problem is passed on as a priority to the deliberative mechanisms. The Deliberative level is provided via a Belief-DesireIntention (BDI) methodology. The deliberation mechanisms are based upon those of Agent Factory (AF) [7, 19]. The architecture provides the agents with the ability to reason based upon beliefs, desires and intentions. The agent structure consists of:

- A Mental State - the agents Belief Set constitutes its mental state. The Belief Set is comprised of Global Beliefs, Local Beliefs and Social Beliefs. The Global Beliefs are comprised of information that is relevant to the agents overall plan and not de- 
pendent on platform. The Local Beliefs contain information pertaining to platform dependent matters. The Social Beliefs are developed based on social interaction between the agent and other socially capable entities. These Beliefs are augmented by information from the Environmental and Reactive Layers.

- Commitment Rules - The Commitment Rules are a core part of the Deliberative Level. These form the rules through which the agent will commit to a particular action based on its current Beliefs Set.

- Commitments - Applying Commitment Rules to Beliefs produce Commitments. They are used to trigger specific actions in the Environmental Layer.

Agent Chameleons are considered as an autonomous, mobile and social entity in the classic multi-agent systems sense. The agent has at any given instance a persona, and associated with a given persona are a given set of capabilities. Knowledge of particular platforms and their capabilities is contained within the Capability Set. The agent has two types of capabilities - internal capabilities, which are independent of platform, and environmental dependent capabilities, that require the agent to possess a particular platform to avail of them. The agent utilises the various capabilities of different platforms through migration.

Social Ability: Due to the very nature of the agent's capacity to migrate from platform to platform with a view to utilising alternate capabilities, the social functionality is embedded throughout the architecture in the form of social beliefs, social preceptors, and social actuators. This ensures agent social ability is platform dependent and is dealt with through the Environmental Layer.

Migration: Successful agent migration necessitates a means of transport between origin and destination devices. Agents locate other platforms through the Agent Factory Registry System, which provides classical white and yellow page services. Agent migration is achieved through cloning. When an agent wants to migrate it informs the destination that it wishes to do so. The destination creates an agent. The mental state of the agent is only then copied and transmitted to the required destination. Upon receipt it is incorporated into the new agent. The old agent is then disposed of and the new agent begins execution.

Mutation: Agent mutation is a core functionality of Agent Chameleons. The embodied form helps the user and other agents in the recognition and subsequent relationship with the entity. In certain circumstances the guise of an agent may need to change to reflect social context and differing roles. The ability for an agent to self determine and opportunistically evolve its form based upon environmental stimuli offers profound opportunities. It is our conjecture that the agent persona is inextricably linked to their associated capabilities, user perception and the user-agent relationship.

\section{Initial Experiments}

By way of illustrating the intelligent apparatus that underpins Agent Chameleons and governs their manifestation and form, we consider three simple experiments agent migration, mutation and survival. Within all three examples the agent behaviour is underpinned by the same decision making process, namely a BDI agent structure. 
Specifically we illustrate how belief adoption drives commitment adoption, which in turn determines agent action.

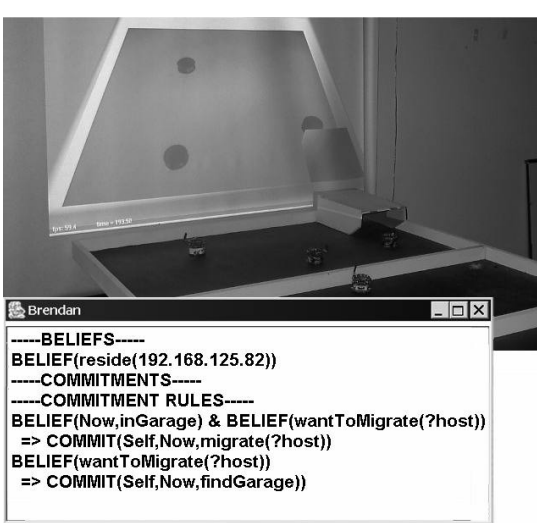

Fig. 2. Pre-migration Mental State

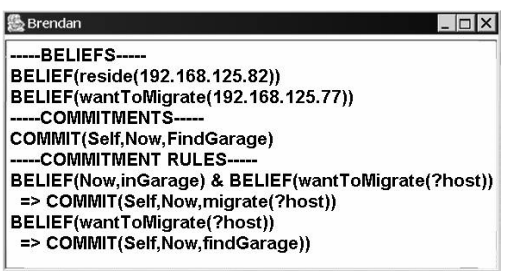

Fig. 3. A Desire to Migrate

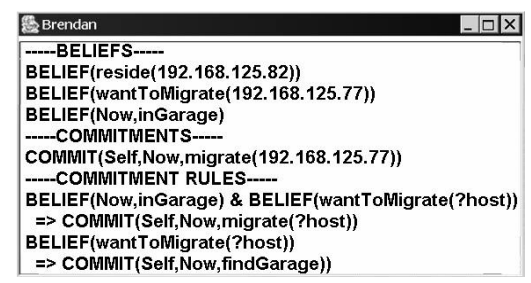

Fig. 4. Commitment to Migration

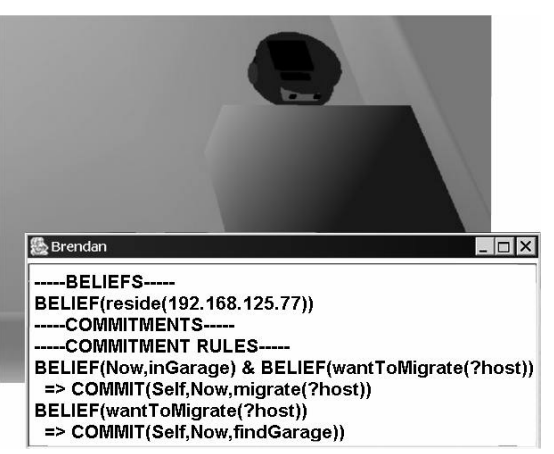

Fig. 5. Migration Complete

Experiment 1: Migration - This demonstrator illustrates the migration of an agent from a physical, real world, robot to a virtual space and vice-versa. In this experiment, a physical world is extended by a virtual world depicted on a computer screen adjoined to the physical world. Small Khepera [12] robots can navigate and explore the desk-mounted world and dock in a robot garage at the edge of the physical world thus removing the physical robot from vision (see Figure 2). Thereafter the robot seamlessly crosses into the virtual world and a virtual robot continues the trajectory of the physical counterpart into the virtual space. Figures 2, 3, 4 and 5, indicate the mental states of the agent at various stages of the experiment. Beliefs, commitments and commitment rules extraneous to the experiment have been omitted from the figures for conciseness. Figure 2 illustrates the pre-migration mental state. Two commitment rules relating to migration are visible. The first indicating if an agent has a desire to migrate it should adopt a commitment to find a garage, and the second indicating that if the agent is in the garage and wants to migrate then it should adopt a commitment to migrate. Figure 3 depicts the activation of the first commitment rule at a subsequent time point, while Figure 4 shows a subsequent belief update relating to a commitment to migrate. Figure 5 illustrates that the migration has now been effected as evidenced by the robot emerging from the virtual garage and by virtue of the agent believing its network address has now changed from 82 to 77 . 
Experiment 2: Mutation - To date various mutations of the virtual agent have been realised. Such mutations are underpinned by commitment rule invocation. For example, in response to a change in the environmental conditions, such as a change in the weather within the virtual environment, the agents may mutate their form. This is illustrated in Figures 7 and 8. When the situation changes in the environment, i.e. when it begins to rain, a belief is generated by a perceptor. Based upon the commitment rules the agent commits to raising the umbrella, triggering an actuator that actually changes the form. In a similar manner Figures 6,7 and 8 illustrate the premutation, mutate adoption and post migration mental states. While the mutation contained within this example constitutes little more than morphing an avatar, mutation is generally much more complex and results in the change of the external or embodied form and the associated capabilities. The capabilities of an agent are inextricably related to the agent form.

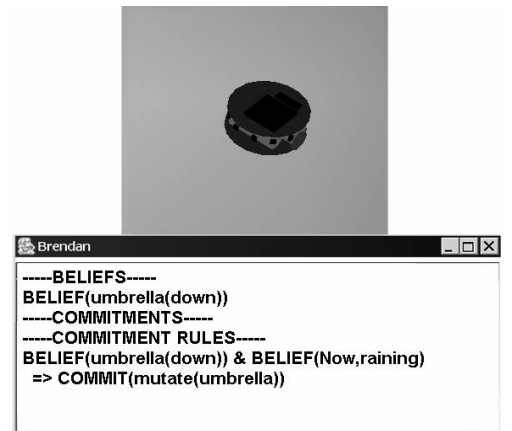

Fig. 6. Pre-mutation Mental State

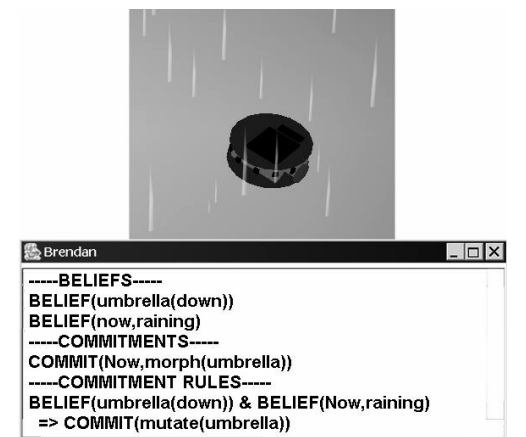

Fig. 7. Commitment to Mutate

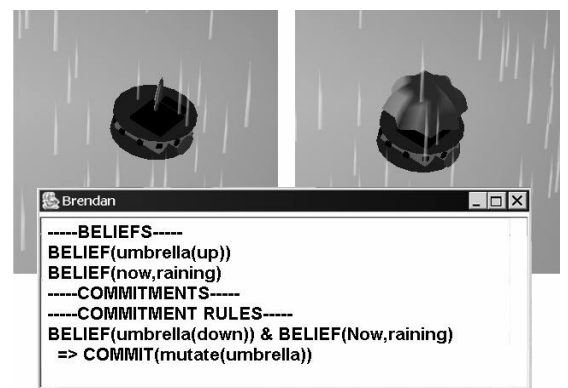

Fig. 8. Post-mutation Mental State

Experiment 3: Survival - The agents used in this research have been attributed basic survival instincts based on the ability of their environment to support their continued operation. For example an agent would have a perceptor monitoring the power supply to its current environment. In the event of low power, the agents' beliefs would be updated accordingly and it will attempt to migrate to protect itself. Whilst a very basic survival behaviour, it is nonetheless indicative of more sophisticated possibilities. 


\section{Conclusion}

Within this paper we have introduced the concept of Agent Chameleons and by way of reference to Lewis Carroll we have provided a fleeting glimpse at the unicorn. Such deductive entities reside within embodied containers and exhibit the key attributes of autonomy, mobility, mutation and evolution. We regard mutation and evolution as higher order attributes synonymous with chameleon agents a new and more sophisticated agent class. The Agent Chameleons Architecture provides the necessary computational support for such migration and mutation. In such nomadic agent environments the degree of social cohesion is typically weaker where agent dynamics produce primarily transient relationships. This is further compounded by the mutation of agent forms, which degrades the visual cues that assist recognition and relationship formation. On-going work is examining the derivation of models of trust reliance and dependence within such nomadic agent communities. We have provided a brief insight into three proof of concept demonstrators that illustrates the fact that mutation and migration are underpinned with the same base BDI architecture.

Acknowledgements. The work undertaken as part of the Agent Chameleons project (http://chameleon.ucd.ie) is a collaborative project between the Department of Computer Science, University College Dublin (UCD) and Media Lab Europe (MLE), Dublin. We gratefully acknowledge the financial support of the Higher Education Authority (HEA) Ireland.

\section{References}

1. Badler, N.I., Phillips, C.B. and Webber, B.L.: Simulating Humans: Computer Graphics, Animation and Control. Oxford University Press, 1993.

2. Benford, S., Schnadelbach, H., Koleva, B., Gaver, B., Schmidt, A., Boucher, A., Steed, A., Anastasi, R., Greenhalgh, C., Rodden, T., and Gellersen, H.: Sensible, Sensable and Desirable: a Framework for Designing Physical Interfaces. Technical Report Equator-03-003, Equator, February 2003.

3. Blumberg, B.M.,: Old Tricks, New Dogs: Ethology and Interactive Creatures. Ph.D Dissertation, MIT, 1996.

4. Brown, B., MacColl, I., Chalmers, M., Galani, A., Randell, C., and Steed, A.: Lessons from the Lighthouse: Collaboration in a Shared Mixed Reality System. In: Proceedings of CHI '2003, New York, January 2003. ACM Press.

5. Cassell, J., Pelachaud, C., Badler, N., Steedman, M., Achorn, B., Becket, T., Douvillle, B., Prevost, S. and Stone, M.: Animated Conversation: Rule-based Generation of Facial Expressions, Jesture \& Spoken Intonation for Multiple Conversational Agents. In: Proceedings of SIGGAPH ' $94,1994$.

6. Cobel, J. and Harbison, K.: MAVE: A multi-agent architecture for virtual environments. In: Proc 11th Int. Conf. on Industrial and Engineering Applications of artificial intelligence and Expert Systems, LNAI, Vol.1415, Springer Verlag, 1998.

7. Collier, R.W.: Agent Factory: An Environment for Prototyping Agents. PhD Thesis, Department of Computer Science, University College Dublin, Dublin, Ireland, 2001. 
8. Collier, R.W., Rooney, C.F.B., O'Donoghue, R.P.S., O'Hare G.M.P.: Mobile BDI Agents. 11th Irish Conference on Artificial Intelligence \& Cognitive Science, University College Galway, Ireland, 2000.

9. Duffy, B.R., O’Hare, G.M.P., Martin, A.N., Bradley, J.F., Schön, B.: Agent Chameleons: Agent Minds and Bodies. The 16th International Conference on Computer Animation and Social Agents - CASA 2003 Rutgers University, New-Brunswick, New Jersey, 7-9 May 2003.

10.Flintham, M., Anastasi, R., Benford, S., et al.: Where on-line meets on-the-streets: Experiences with mobile mixed reality games. CHI 2003, to appear.

11.Johnson, L. and Rickel, J.: Virtual humans for team training in VR. Proc. 9th World Conference on AI in Education, July 1999, pp578-585.

12.K-Team: Khepera. http://www.k-team.com

13.Pérez, P., Méndez G. and Antonio, A.: mIVA: Why to Use Mobile Agents in Virtual Environments and Wireless Devices. ECAI 2002, 15th European Conference on Artificial Intelligence. Workshop W6, Artificial Intelligence in Mobile System, Lyon, France. July 2002.

14. Norman, T.J., Jennings, N.R.: Constructing a virtual training laboratory using intelligent agents. Int Journal of Continuous Engineering and Life-Long Learning, 2000.

15.O'Hare, G.M.P.: Agents, Mobility and Virtuality: A Necessary Synergy. In: Proceedings of International ICIS Symposium on Multi-Agents and Mobile Agents in Virtual Organizations and E-Commerce (MAMA '2000), December 11-13, 2000, Wollongong, ICSC Academic Press, ISBN 3-906454-24-X, 2000.

16. O'Hare, G.M.P., Sewell, K., Murphy, A. and Delahunty, T.: ECHOES: An Immersive Training Experience. In: Proceedings of International Conference on Adaptive Hypermedia and Adaptive Web-based Systems (AH2000), Springer Verlag.

17. O'Hare, G.M.P., Duffy, B.R.: Agent Chameleons: Migration and Mutation within and between Real and Virtual Spaces. The Society for the Study of Artificial Intelligence and the Simulation of Behaviour AISB 2002, Imperial College, England, April 3-5, 2002.

18.O'Hare, G.M.P., Duffy, B.R. \& Collier, R.W.: NEXUS - A Singularity Between the Real and the Virtual. HEA Research Proposal, Department of Computer Science, University College Dublin, Belfield, Dublin 8, Ireland. 2003.

19. O'Hare, G.M.P., O'Hare, P.T. and Lowen, T.D.: Far and A WAY: Context Sensitive Service Delivery through Mobile Lightweight PDA hosted Agents. Proceedings of 15th International Florida Artificial Intelligence (FLAIRS) Conference. AAAI Press. Pensacola, Florida. May 14th-16th, 2002.

20.O'Hare, G.M.P., Duffy, B.R., Bradley, J.F. and Martin, A.N.: Agent Chameleons: Moving Minds from Robots to Digital Information Spaces. Proceedings of Autonomous Minirobots for Research and Edutainment (AMiRE) 2003 Brisbane, Australia, February 18-21 2003.

21.Reilly, W.S.: Believable Social and Emotional Agents, PhD dissertation, CMU 1996.

22. Steels, L.: Building Agents with Autonomous Behaviour Systems. The 'artificial life' route to 'artificial intelligence'. Building situated embodied agents. Lawrence Erlbaum Associates, New Haven. 1994

23. Sumi, Y., Mase, K.: AgentSalon: Facilitating Face to-Face Knowledge Exchange through Conversations Among Personal Agents. Fifth International Conference on Autonomous Agents (Agents 2001), pp.393

24.Vosinakis, S., Anastassakis, G. and Panayiotopoulos, T.: DIVA: Distributed Intelligent Virtual Agents. Proc. VA99, Workshop on Intelligent Virtual Agents, at UK, VRSIG, Sept 1999. 